\title{
一锅法合成基于丁二炔-苯环冠醚的[2]轮烷
}

\author{
陈木娟郭浩玉周松根陈 坤蒋腊生* \\ (华南师范大学化学与环境学院 广州 510006)
}

\begin{abstract}
摘要 以丁二炔-苯环冠醚作为大环化合物, 以带有 4,4'-联吡啶正离子结构的线性分子作为半封端分子, 通过简便的一 锅法合成一种新的 [2]轮烷分子, 产率为 $24 \%$. 经高分辨质谱、核磁共振氢谱、核磁共振碳谱等手段确认了丁二炔一苯环 冠醚大环与带有两个 4,4'-联吡啶二正离子的线性封端分子组装形成[2]轮烷结构, 且丁二炔-苯环冠醚在线性分子的两 个识别点间作往返运动.

关键词 丁二炔-苯环冠醚; 一锅法; 合成; [2]轮烷
\end{abstract}

\section{One-Pot Synthesis of A New [2]rotaxane Based on the Phenylene-Diacetylene Crown Ether}

\author{
Chen, Mujuan Guo, Haoyu Zhou, Songgen Chen, Kun Jiang, Lasheng* \\ (School of Chemistry and Environment, South China Normal University, Guangzhou 510006)
}

\begin{abstract}
A new [2] rotaxane was synthesized via one-pot method, based on the assembly of the phenylene-diacetylene crown ether (acted as ring molecule) and the paraquat derivative (acted as semi-axle molecule). The HR-MS, ${ }^{1} \mathrm{H}$ NMR and ${ }^{13} \mathrm{C}$ NMR spectra have confirmed that one phenylene-diacetylene crown ether ring threaded though the axle molecule forming the corresponding [2]rotaxane, and the macrocyclic ring moves from one 4,4'-bipyridinium station to another 4,4'-bipyridinium station.
\end{abstract}

Keywords phenylene-diacetylene crown ether; one-pot method; synthesis; [2]rotaxane

超分子化学是当前化学学科的热点研究领域之一, 各种超分子体系层出不穷 ${ }^{[1]}$. 其中轮烷是一种由环状分 子与哑铃状线性分子通过非共价键作用形成的超分子 体系, 由于其新奇的互锁型结构特性, 使其在分子机器 设计以及功能材料开发等方面得到了广泛的关注 ${ }^{[2]}$. 迄 今合成轮烷分子的方法多种多样, 绝大多数方法都是在 溶液中利用模板合成, 即利用大环分子和亚铃状线性分 子的相互识别、组装形成的非共价键作用合成. 目前被 广泛应用的非共价键作用主要有氢键、离子配体络合作 用和 $\pi-\pi$ 堆积作用等 ${ }^{[3]}$.

根据组装过程的差异, 轮烷分子的合成方式一般有 五种: 穿线封端法(treading) ${ }^{[4]}$ 、夹套法(clipping) $)^{[5]}$ 、滑入 法 (slipping) ${ }^{[6]}$ 、穿 线 胀大法 (threading-followed-byswelling $)^{[7]}$ 和诱捕法(trapping $)^{[8]}$. 诱捕法是由 Fritz Vögtle 等提出的, 是先使封端线性分子片段与环状分子形
成环状配合物后，诱使另一线形分子片段与其在大环空 腔内反应，最终生成轮烷分子的一种合成轮烷的方法. 1999 年, 他们利用对三苯甲基-苯酚离子作为塞子与酰 胺大环在氢键作用下形成络合物后, 酚氧离子再与带苠 澳基团的另一封端线性分子发生亲核取代反应最终生 成轮烷 ${ }^{[8 a]}$. 与前面四种合成方式不同的是诱捕法在线性 分子形成之前大环化合物与线性分子之间没有发生穿 入或环绕的过程，线性分子是在大环化合物与线性分子 片段形成的络合物的诱导下发生键合的，最终才生成轮 烷. 2007 年, Hirose 等 ${ }^{[9]}$ 利用酰氯发生胺解反应分别使 3,5-二甲基苯甲酰氯和 3,5-二硝基苯甲酰氯与酚羟基冠 醚大环形成络合物，再与含芐胺基团塞子反应直接生产 轮烷，此合成路线利用了大环分子与线性分子上的酰胺 键形成的氢键作用. 2013 年, Winn 等 ${ }^{[10]}$ 利用含乙炔基塞 子和含叠氮基塞子在一价铜离子的催化下，使两者在大

\footnotetext{
*E-mail: jianglsh@scnu.edu.cn

Received June 7, 2014; revised July 19, 2014; published online August 11, 2014.

Project supported by the National Natural Science Foundation of China (No. 21072066) and the Key Project on the Integration of Industry, Education and Research of Guangdong Province (No. 2012B090700003).

国家自然科学基金(No. 21072066)和广东省省部产学研基金(No. 2012B090700003)资助项目。
} 
环空腔内发生炔一叠氮环加成反应生成线性分子, 使线 性分子与环状化合物在离子络合作用下生成[2]轮烷. 上述研究显示了在氢键或金属离子络合作用的模板作 用下，大环化合物与线性分子片段两者即使在形成轮烷 之前没有发生环绕或穿入，也能够有效的识别组装并通 过共价键的形成最终生成轮烷分子. 受这些合成过程的 启示，本文设计了一种基于环状化合物与线性分子之间 $\pi-\pi$ 堆积作用的合成轮烷的路线, 其中以丁二炔-苯环冠 醚作为大环分子，4,4-联吡啶正离子作为线性分子片段, 一锅法合成轮烷分子.

丁二炔-苯环冠醚是我们课题组合成的一种含有丁 二炔结构的冠醚大环，以多甘醇为起始原料，采用 Egliton 炔炔偶联关环反应，具有合成步骤短，总收率高 等优点, 并通过实验手段证明丁二炔一苯环冠醚能够与 $N, N^{\prime}$-二甲基-4,4'-联吡啶盐在 $\pi-\pi$ 堆积和氢键的协同作用 下在溶液和固态中形成稳定的 $[n]$ 准轮烷 ${ }^{[11]}$, 并利用夹 套法(clipping)成功制备基于丁二炔-苯环冠醚大环与联 吡啶环番的[2]索烃分子(图 1) ${ }^{[12]}$. 因此, 我们基于诱捕 法合成轮烷分子的思路, 设计一锅法合成丁二炔-苯环 冠醚与带有 4,4'-联吡啶正离子识别点的哑铃状线性分 子的轮烷分子 3 , 合成路线如 Scheme 1 所示.
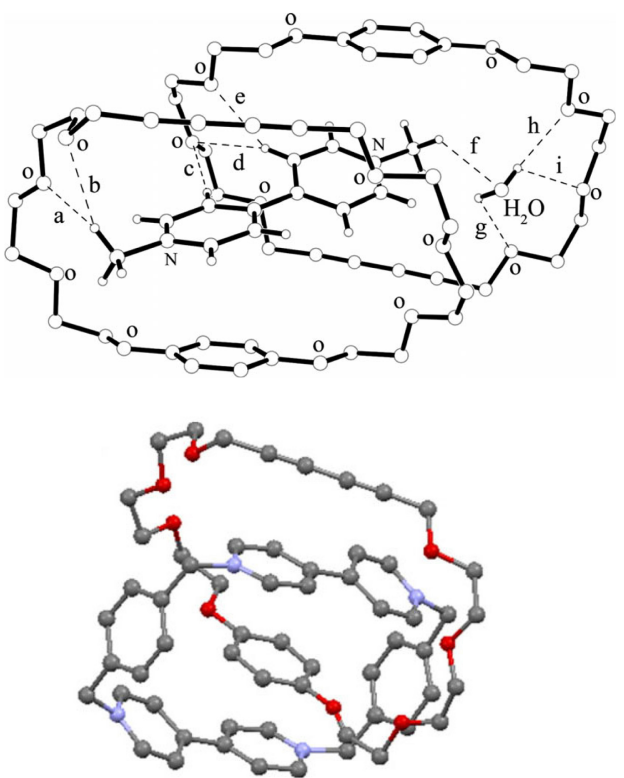

图 1 基于丁二炔-苯环冠醚的准轮烷分子 ${ }^{[11]}$ 和索烃分子 ${ }^{[12]}$

Figure 1 Structure of [3]pseudorotaxane-like complexe ${ }^{[11]}$ and [2] catenane ${ }^{[12]}$ based on macrocycle $\mathbf{1}$

\section{1 结果与讨论}

\section{1 [2]轮烷的合成}

二芳基大环冠醚由于具有富电子的芳基和容易形<smiles>CC(C)(C)c1ccc(C(c2ccccc2)(c2ccc(OCCOCCOc3ccc(C[n+]4ccc(-c5ccncc5)cc4)cc3)cc2)c2ccc(C(C)(C)C)cc2)cc1</smiles>

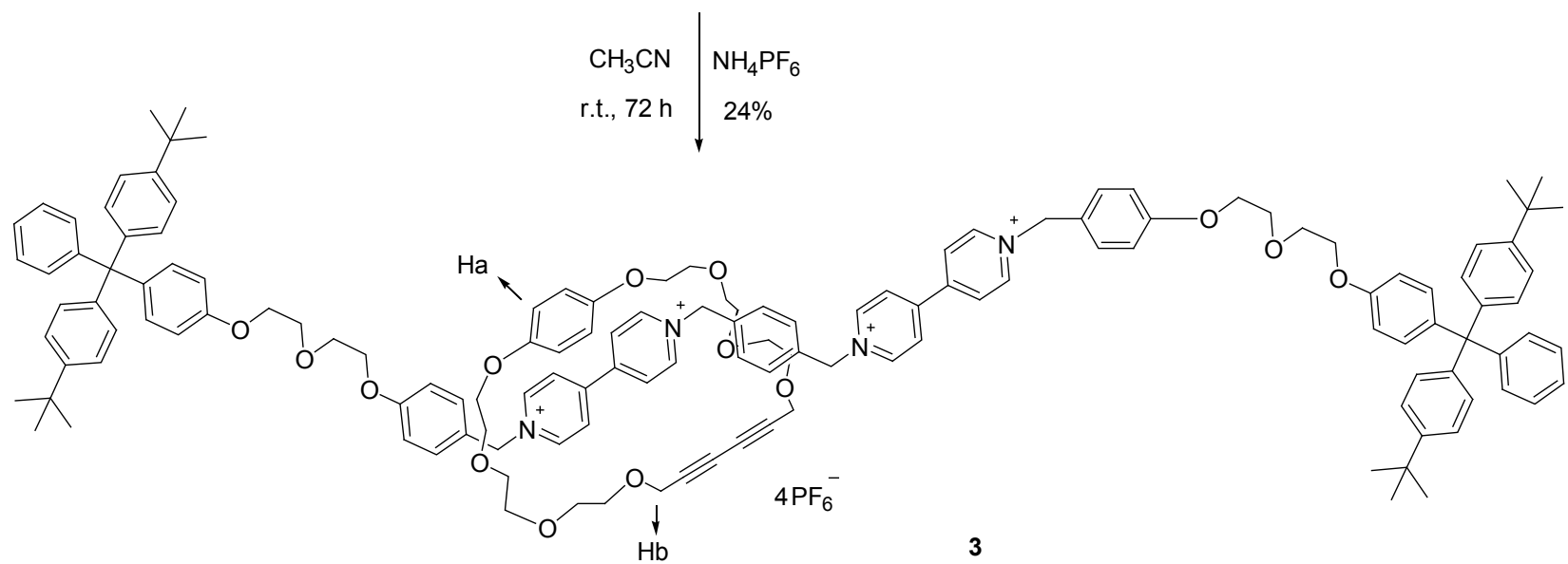

Scheme 1 
成氢键的烷氧基单元而被广泛应用于轮烷、索烃及相关 分子机器的组装与制备, 如 34-冠-10 $10^{[13]}$ 等. 34-冠-10 与 4,4'-联吡啶正离子盐之间有较强的 $\pi-\pi$ 堆积作用和氢键 作用, 两者能够识别组装形成不同结构的超分子体系. 1996 年, Stoddart 课题组 ${ }^{[6]}$ 曾经在超高压条件下利用穿 线法和滑入法, 合成了基于 34 -冠-10 与联吡啶正离子识 别组装的 $[n]$ 轮烷. 穿线法是在高压条件下以联吡啶正 离子作为线性分子, 在 DMF 溶液中与 34-冠-10 (4 equiv.) 和封端塞子反应 $2 \mathrm{~d}$, [2]轮烷, [3] 轮烷的收率分别 为 $5 \%$ 和 $33 \%$; 滑入法是在加热的条件下, 使已经封端 的线性分子与 34-冠-10 (4 equiv.)反应 $10 \mathrm{~d}$, [2]轮烷和 [3] 轮烷的收率分别为 $31 \%$ 和 $8 \%$. 这种两种合成轮烷的 方法涉及的实验步骤都比较多, 且反应条件都比较严 格, 轮烷的总收率都不高. 由于丁二炔结构在加热的条 件下发生聚合反应, 所以不能利用滑入法合成基于丁二 炔-苯环冠醚的轮烷分子. 因此, 我们根据丁二炔-苯环 冠醚的结构特征提出新的合成路线一一锅法, 这是一 种在常温常压下一步合成轮烷的方法. 具体合成路线是 先使带有联吡啶正离子识别点的线性分子片段与冠醚 大环组装形成络合物, 之后诱捕另一线性分子片段反应 生成线性分子, 最后形成轮烷分子. 我们通过在塞子分 子 4 上引入官能团芐溴, 将其与 4,4'-联吡啶反应得到带 塞子的单正离子 2 (Scheme 3). 之后将其与对苯二芐溴 以及丁二炔-苯环冠醚在乙腈溶剂中, 在室温氮气保护 的条件下摚拌反应 $72 \mathrm{~h}$, 经柱层析分离, 离子交换后得 到红色的固体[2]轮烷分子 3. 在丁二炔-苯环冠醚的投 放量也是线性分子的 4 equiv. 的条件下, [2]轮烷的收率 为 $24 \%$, 整个合成步骤简单.

在此实验过程中我们没有分离得到[3]轮烷, 我们 推测在本文的实验条件下, 由于 $4,4^{\prime}$-联吡啶二正离子与 丁二炔-苯环冠醚 $\mathbf{1}$ 的识别能力更强, 因此, 单正离子 $\mathbf{2}$ 优先与对苯二芐溴反应生成 4,4'-联吡啶二正离子盐, 再 与大环化合物 1 组装, 形成稳定性更高的环状化合物的 络合物, 再 “诱捕” 另一线性分子片段反应, 最后得到 目标产物[2]轮烷 3, 如 Scheme 2 所示.

\section{2 [2]轮烷的结构解析}

轮烷分子的结构可以通过质谱和核磁实验表征确 定. 在高分辨质谱图中(图 2), $m / z$ 峰 2551.04386 对应的 是[2]轮烷分子 $\left[3-\mathrm{PF}_{6}\right]^{+}$(calcd for $\mathrm{C}_{140} \mathrm{H}_{154} \mathrm{~F}_{18} \mathrm{~N}_{4} \mathrm{O}_{14} \mathrm{P}_{3}$ 2551.04205); $\mathrm{m} / \mathrm{z}$ 峰 1203.04197 对应的是[2]轮烷分子 $\left[3-2 \mathrm{PF}_{6}\right]^{2+}$ (calcd for $\mathrm{C}_{140} \mathrm{H}_{154} \mathrm{~F}_{12} \mathrm{~N}_{4} \mathrm{O}_{14} \mathrm{P}_{2}$ 2406.07787); $\mathrm{m} / \mathrm{z}$ 峰 625.36789 和 817.87315 分别是 $[\mathbf{5}-\mathrm{Br}]^{+}$(calcd for $\mathrm{C}_{44} \mathrm{H}_{49} \mathrm{O}_{3}$ 625.36817)和 $\left[3-5-3 \mathrm{PF}_{6}\right]^{2+}$ 离子峰(calcd for $\mathrm{C}_{96} \mathrm{H}_{105} \mathrm{~F}_{6} \mathrm{~N}_{4} \mathrm{O}_{11} \mathrm{P}$ 1635.74552), 对应于[2]轮烷分子 3 其
中一个联吡啶识别点上的吡啶 $\mathrm{N}$ 与塞子基团上的芐亚 甲基处断裂所形成的两个质谱峰. 高分辨质谱图表明利 用一锅法成功合成获得了丁二炔-苯环冠醚与带有 4,4'联吡啶二正离子识别点的线性分子组装而成的 [2]轮烷 3.

核磁数据进一步说明了 [2]轮烷分子的形成. 在丁 二炔-苯环冠醚 $\mathbf{1}$ 与[2]轮烷分子 $\mathbf{3}$ 的氢谱对照图中可以 看出(图 3), 丁二炔-苯环冠醚组装成轮烷后, 大环上苯 环氢原子 $\mathrm{Ha}$ 化学位移由 $\delta 6.89$ 移动到了 $\delta 6.17$, 向高场 移动了 $\delta 0.72$, 大环上炔丙位的氢 $\mathrm{Hb}$ 由 $\delta 4.30$ 移动到 3.94 , 亦向高场移动了 $\delta 0.36$, 表明 4,4'-联吡啶正离子识 别点已经穿入丁二炔-苯环冠醚大环内, 氢原子 $\mathrm{Ha}, \mathrm{Hb}$ 处于联吡啶盐的屏蔽区, 所以化学位移向高场移动. 丁 二炔-苯环冠醚 1 与带有 4,4'-联吡啶正离子识别点的线 性分子在 $\pi-\pi$ 堆积作用的驱使下发生识别组装形成[2] 轮烷.

在[2]轮烷 $\mathbf{3}$ 的核磁氢谱图上(图 3, B), [2]轮烷 3 各 个位置上的氢原子的化学位移仅出现一个明确的谱值, 如联吡啶盐上 $\alpha 、 \beta$ 位上的氢. 核磁结果没有出现一个与 丁二炔-苯环冠醚大环作用后的氢原子核磁谱峰以及一 个 “裸露” 在外的氢原子核磁谱峰, 这说明丁二炔-苯环 冠醚 $\mathbf{1}$ 由于两端塞子的堵塞作用，被限制于线性分子之 内, 在两个吡啶盐识别点位置间作穿梭运动, 且大环在 线性分子上的穿梭运动频率大于核磁时间分辨率，所以 核磁检测到的是吡啶盐与大环作用后的一个平均化学 位移值, 这就形成了超分子化学领域中的一类分子机 器——分子梭(Shuttle), 如图 4.

质谱和核磁数据表明利用一锅法成功制备了基于 丁二炔-苯环冠醚的 [2]轮烷, 并且从核磁数据上可知在 室温条件下大环分子在线性分子上的两个 4,4'-联吡啶 二正离子识别点间做自主的往返运动.

\section{2 结论}

本文利用一锅法成功合成了基于丁二炔-苯环冠醚 大环与 4,4'-联吡啶二正离子识别组装生成的 [2]轮烷分 子, 并通过高分辨质谱、核磁氢谱、碳谱等分析手段对 其进行了结构表征. 实验事实表明即使其中一个芳香基 团被丁二炔结构取代, 丁二炔-苯环冠醚依然能够与联 吡啶正离子在 $\pi-\pi$ 堆积作用的驱使下识别组装形成[2] 轮烷超分子体系. 此外, 在芳基冠醚上引入丁二炔结构, 不仅优化了大环冠醚的合成路线, 而且丁二炔结构在光 照或加热条件下能够发生聚合, 形成一类在光电材料、 生物化学传感器等领域应用前景广阔的含共轭 $\pi$ 键的聚 合物(Polydiacetylene, PDA). 若能把本文所合成[2]轮 
<smiles>CC(C)(C)c1ccc(C(c2ccccc2)(c2ccc(OCCOCCOc3ccc(C[n+]4ccc(-c5ccncc5)cc4)cc3)cc2)c2ccc(C(C)(C)C)cc2)cc1</smiles><smiles>BrCc1ccc(CBr)cc1</smiles><smiles>CC(C)(C)c1ccc(C(c2ccccc2)(c2ccc(OCCOCCOc3ccc(C[n+]4ccc(-c5cc[n+](Cc6ccc(CBr)cc6)cc5)cc4)cc3)cc2)c2ccc(C(C)(C)C)cc2)cc1</smiles><smiles></smiles>

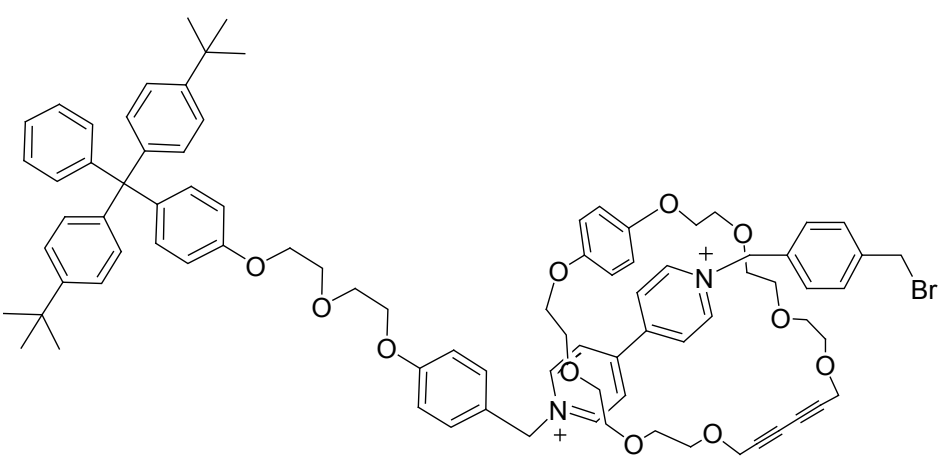<smiles>CCCCCCC(c1ccc(OCCOCCOc2ccc(C[n+]3ccc(-c4ccncc4)cc3)cc2)cc1)c1ccc(C(C)(C)C)cc1</smiles>

$2 \mathrm{PF}_{6}^{-}$<smiles>COCCOCCOc1ccc(C(c2ccccc2)(c2ccc(C(C)(C)C)cc2)c2ccc(C(C)(C)C)cc2)cc1</smiles>

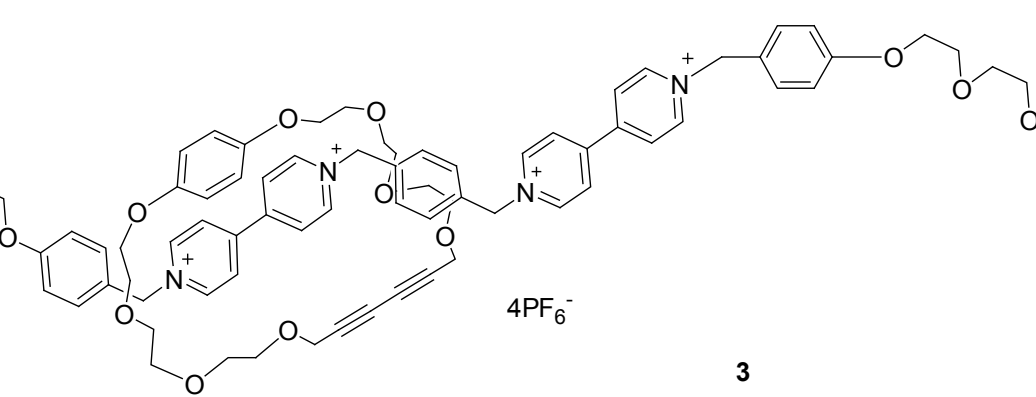<smiles>COc1ccc(C(c2ccccc2)(c2ccc(C(C)(C)C)cc2)c2ccc(C(C)(C)C)cc2)cc1</smiles>

Scheme 2

烷体系引入共轭聚合物的合成上无疑是非常有意思的, 对开发新型功能材料也具有基础研究价值和潜在应有 价值, 相关的一些研究正在进行, 而对于[2]轮烷分子在 常温常压条件下作自主穿梭运动的讨论研究也在进行 中.

\section{3 实验部分}

\section{1 仪器与试剂}

所用试剂均为市售分析纯; 柱层析硅胶为青岛海洋 化工厂生产，未经活化; NMR 采用美国 varian NMR 


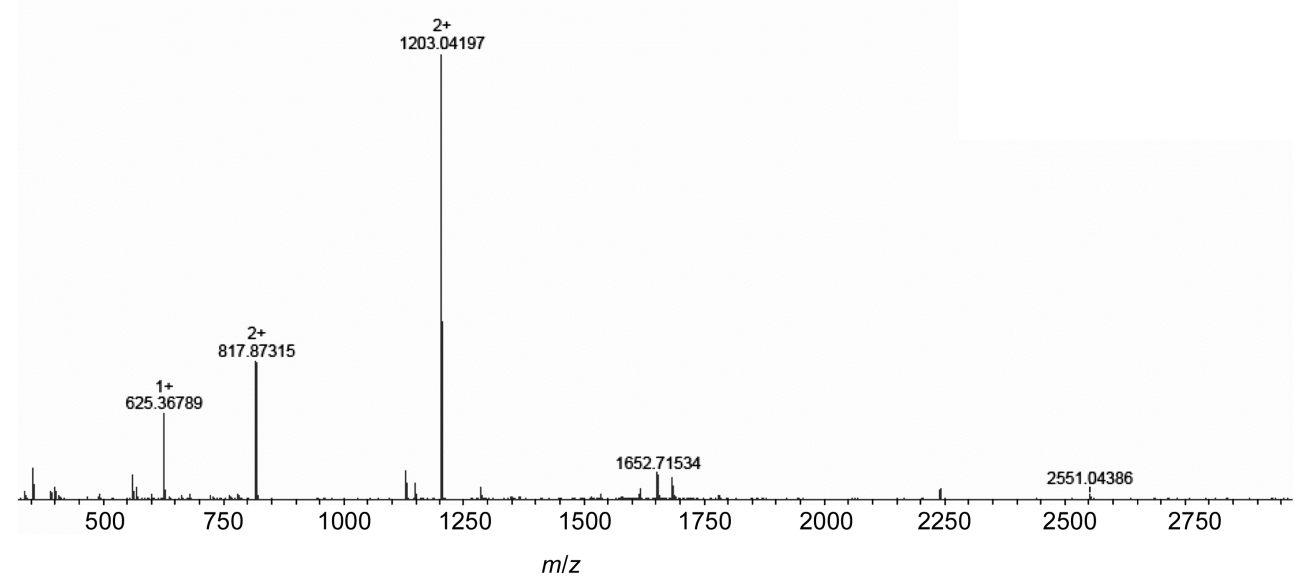

图 2 [2]轮烷 3 高分辨质谱图

Figure 2 HR-MS spectrum of the [2] rotaxane 3

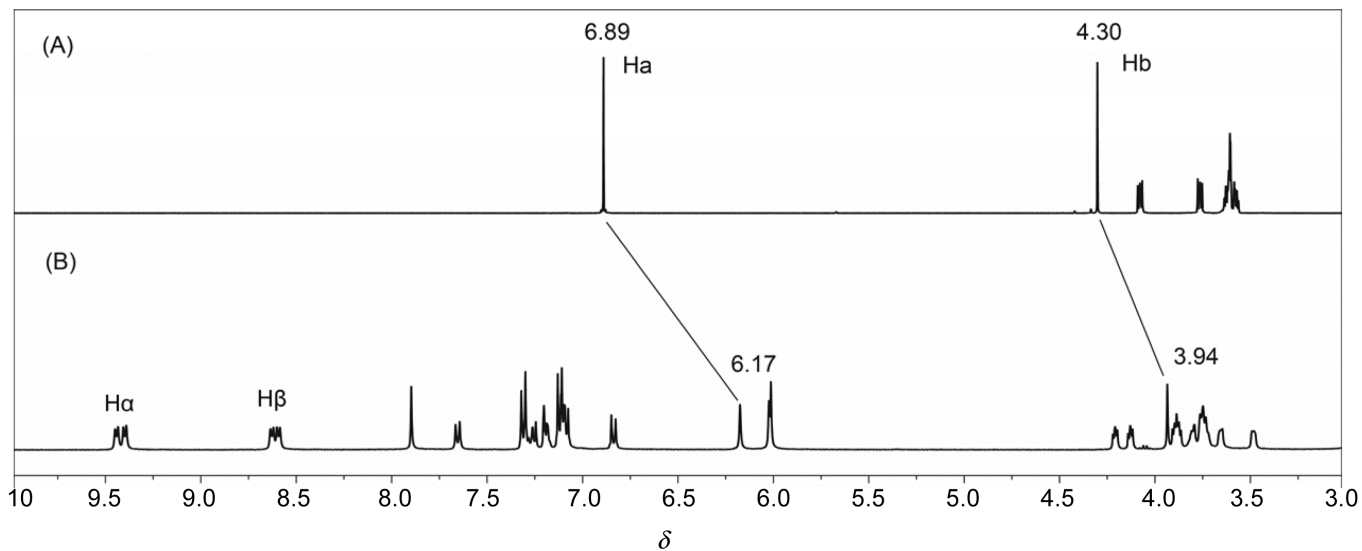

图 3 大环化合物 $\mathbf{1}(\mathrm{A})$ 与[2]轮烷 $3(\mathrm{~B})$ 部分 ${ }^{1} \mathrm{H}$ NMR 化学位移对比

Figure 3 Partial ${ }^{1} \mathrm{H}$ NMR spectra $\left(400 \mathrm{MHz}, \mathrm{CD}_{3} \mathrm{COCD}_{3}\right)$ of (A) macrocycle 1 and (B) [2]rotaxane 3

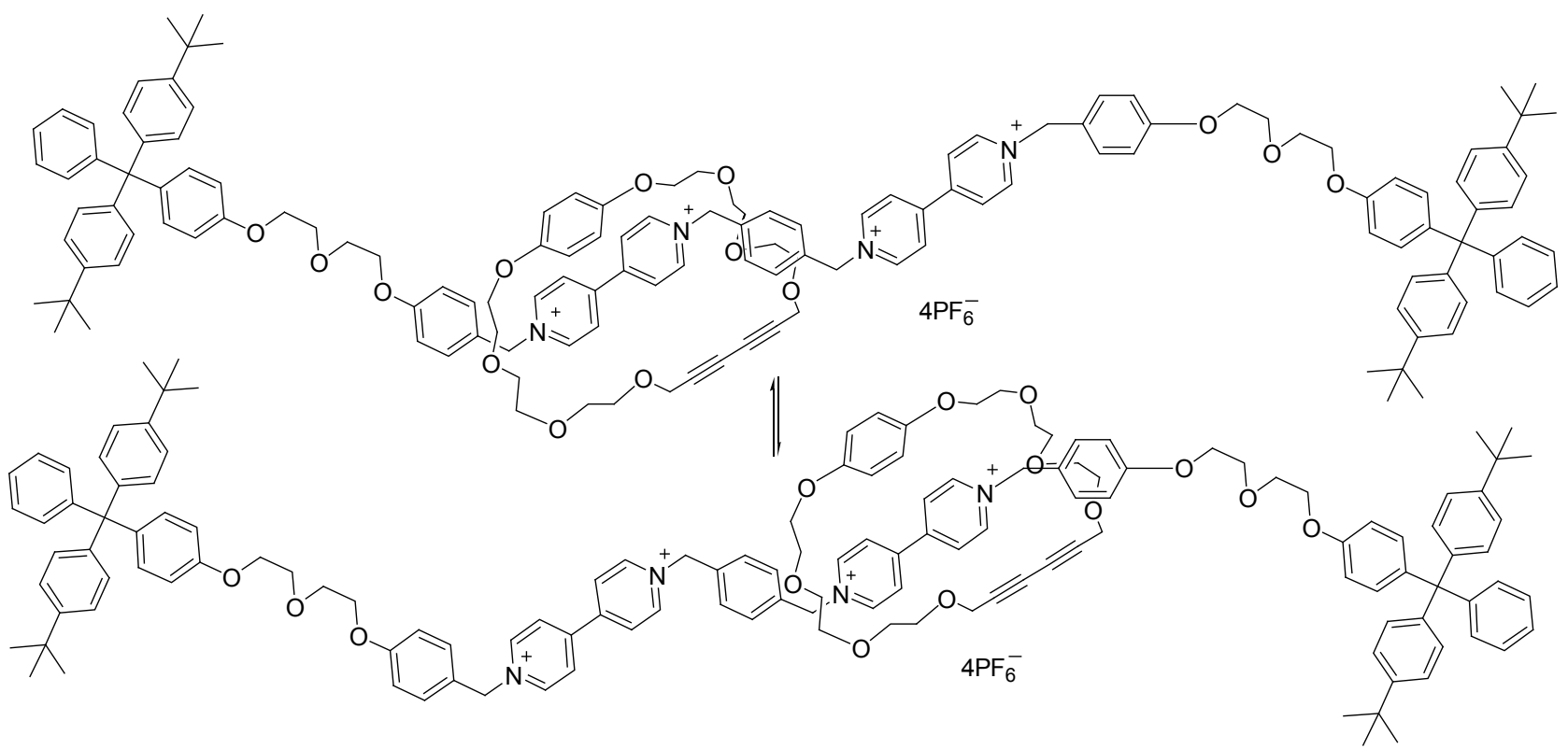

图 4 基于丁二炔-苯环冠醚的[2]轮烷分子梭

Figure 4 [2]rotaxane shuttle based on the phenylene-diacetylene crown ether 
system $400 \mathrm{MHz}$ 核磁共振仪, 溶剂为気代丙酮 $\left(\mathrm{CD}_{3} \mathrm{COCD}_{3}\right)$; LR-MS 采用 LCQ Advantage 液质联用仪, ESI 源; HR-MS 在北京大学分析测试中心测, 采用 Bruker Apex IV FTMS, ESI 源; 熔点测定采用 X-5 显微 熔点测定仪, 温度计未校正.

\section{2 实验方法}

\section{2 .1 化合物 $\mathbf{1}$ 的合成}

化合物 1 参考文献[11]合成.

\section{2 .2 化合物 $\mathbf{5}$ 的合成}

化合物 4 的合成参考文献[6]. 在氮气的保护下，往 干燥的两口烧瓶中加入化合物 $4(0.49 \mathrm{~g}, 0.76 \mathrm{mmol})$, 四 氢呋喃 $5 \mathrm{~mL}$. 用注射器向反应瓶中注入 $1.16 \mathrm{~mL} \mathrm{PBr}_{3}$, 室温下搅拌反应 $18 \mathrm{~h}$. 薄层层析跟踪反应进程 $\left[V\left(\mathrm{CH}_{2} \mathrm{Cl}_{2}\right): V(\right.$ P.E. $\left.)=2: 1\right]$, 直至化合物 4 原料点完全 消失. 反应结束后, 向反应瓶中加入 $15 \mathrm{~mL}$ 的冰水, 冰 浴下快速振荡, 以便使 $\mathrm{PBr}_{3}$ 水解完全. 向反应混合液中 加入 $30 \mathrm{~mL}$ 的 $\mathrm{CHCl}_{3}$, 分出有机层, 水层用 $\mathrm{CHCl}_{3}(30$ $\mathrm{mL} \times 2$ ) 快速萃取. 合并有机层, 无水硫酸镁干燥, 过滤, 蒸馏除去溶剂得到白色固体 ${ }^{[14]}$, 直接用于下一步合成.

\section{2 .3 化合物 $\mathbf{2}$ 的合成}

在氮气保护和磁力摚拌下，向盛有 $30 \mathrm{~mL}$ 乙腈溶液 的反应瓶中加入 $4,4^{\prime}$-联吡啶 $(0.40 \mathrm{~g}, 2.56 \mathrm{mmol})$, 将溶有 $5(0.45 \mathrm{~g}, 0.64 \mathrm{mmol})$ 的 $20 \mathrm{~mL}$ 的乙腈溶液在 $363 \mathrm{~K}$ 的温 度下从恒压漏斗缓慢滴加至反应瓶，滴加完毕，反应 48 $\mathrm{h}$ 后, 浓缩, 柱色谱分离 $\left[V\left(\mathrm{CH}_{3} \mathrm{OH}\right): V\left(\mathrm{CH}_{3} \mathrm{CN}\right): V(2\right.$ $\left.\left.\mathrm{mol} / \mathrm{L} \mathrm{NH}_{4} \mathrm{Cl}\right)=70: 30 ： 9\right]$, 将产品溶于丙酮水溶液 $(V: V=1: 1)$, 用六氟磷酸铵交换, 再浓缩除去丙酮, 得含白色固体悬浊液, 抽滤, 将所得滤饼真空干燥, 得 白色固体化合物 $\mathbf{2}^{[14]}$, 两步总产率 76\%. ${ }^{1} \mathrm{H}$ NMR (400 $\left.\mathrm{MHz}, \mathrm{CDCl}_{3}\right) \delta: 9.62(\mathrm{~s}, 2 \mathrm{H}), 8.84(\mathrm{~s}, 2 \mathrm{H}), 8.17(\mathrm{~s}, 2 \mathrm{H})$, $7.60(\mathrm{~d}, J=8.0 \mathrm{~Hz}, 4 \mathrm{H}), 7.25 \sim 7.11(\mathrm{~m}, 9 \mathrm{H}), 7.08$ (dd, $J=$ $8.2,5.6 \mathrm{~Hz}, 6 \mathrm{H}), 6.91(\mathrm{~d}, J=8.2 \mathrm{~Hz}, 2 \mathrm{H}), 6.76(\mathrm{~d}, J=8.7$ $\mathrm{Hz}, 2 \mathrm{H}), 6.23$ (s, 2H), 4.15 4.06 (m, 4H), 3.94 3.85 (m, $4 \mathrm{H}), 1.27(\mathrm{~s}, 18 \mathrm{H})$.

3.2.4 化合物 $\mathbf{3}$ 的合成

在氮气保护和磁力搅拌下，向盛有 $4.2 \mathrm{~mL}$ 乙腈溶 液的反应瓶中依次加入 $2(0.18 \mathrm{~g}, 0.19 \mathrm{mmol})$, 对苯二芐 溴 $(0.026 \mathrm{~g}, 0.097 \mathrm{mmol})$, 丁二炔-苯环冠醚 1 (0.21 g, $0.46 \mathrm{mmol})$, 室温反应 $72 \mathrm{~h}$ 后, 浓缩, 将粗产品直接柱 色谱分离 $\left[V\left(\mathrm{CH}_{3} \mathrm{OH}\right): V\left(\mathrm{CH}_{3} \mathrm{CN}\right): V\left(2 \mathrm{~mol} / \mathrm{L} \mathrm{NH}_{4} \mathrm{Cl}\right)=\right.$ $70: 30: 1.5]$, 将所得产品溶于丙酮水溶液 $(V: V=1:$ $1)$, 用六氟磷酸铵交换, 再浓缩除去丙酮, 得含红色固 体悬浊液，抽滤，将所得滤饼真空干燥，得红色固体 63 $\mathrm{mg}$, 产率 24\%. m.p. $145{ }^{\circ} \mathrm{C}$; ${ }^{1} \mathrm{H}$ NMR $(400 \mathrm{MHz}$, $\left.\mathrm{CD}_{3} \mathrm{COCD}_{3}\right) \delta: 9.42(\mathrm{dd}, J=17.2,6.2 \mathrm{~Hz}, 8 \mathrm{H}), 8.61(\mathrm{dd}$, $J=13.7,6.5 \mathrm{~Hz}, 8 \mathrm{H}), 7.90$ (s, 4H), 7.65 (d, $J=8.6 \mathrm{~Hz}$, $4 \mathrm{H}), 7.39 \sim 7.03(\mathrm{~m}, 34 \mathrm{H}), 6.84(\mathrm{~d}, J=8.9 \mathrm{~Hz}, 4 \mathrm{H}), 6.17$ (s, 4H), $6.02(\mathrm{~s}, 4 \mathrm{H}), 6.01(\mathrm{~s}, 4 \mathrm{H}), 4.24 \sim 4.19(\mathrm{~m}, 4 \mathrm{H})$, $4.16 \sim 4.10(\mathrm{~m}, 4 \mathrm{H}), 3.94(\mathrm{~s}, 4 \mathrm{H}), 3.92 \sim 3.70(\mathrm{~m}, 24 \mathrm{H})$, $3.68 \sim 3.63(\mathrm{~m}, 4 \mathrm{H}), 3.51 \sim 3.45(\mathrm{~m}, 4 \mathrm{H}), 1.29$ (s, 36H); ${ }^{13} \mathrm{C}$ NMR $\left(100 \mathrm{MHz}, \mathrm{CD}_{3} \mathrm{COCD}_{3}\right) \delta: 160.27,156.91$, $152.07,148.46,148.33,147.40,145.84,145.51,144.15$, $139.23,135.15,131.92,131.34,130.80,130.58,130.51$, $127.34,126.46,126.28,125.76,125.21,124.20,115.34$, $114.69,113.19,104.99,70.44,70.17,69.86,69.58,69.39$, $67.71,67.46,67.41,67.29,64.24,64.03,63.38,33.95$, 30.72; LR-MS (ESI) $\mathrm{m} / z(\%): 625.68\left([5-\mathrm{Br}]^{+}, 100\right)$, $781.45\left([2]^{+}, 53\right), 1203.07\left(\left[\mathrm{M}-2 \mathrm{PF}_{6}\right]^{2+}, 13\right) ;$ HRMS (ESI) calcd for $\mathrm{C}_{140} \mathrm{H}_{154} \mathrm{~F}_{12} \mathrm{~N}_{4} \mathrm{O}_{14} \mathrm{P}_{2}\left(\left[\mathrm{M}-2 \mathrm{PF}_{6}\right]^{2+}\right)$ 2406.07787, found 1203.04197.

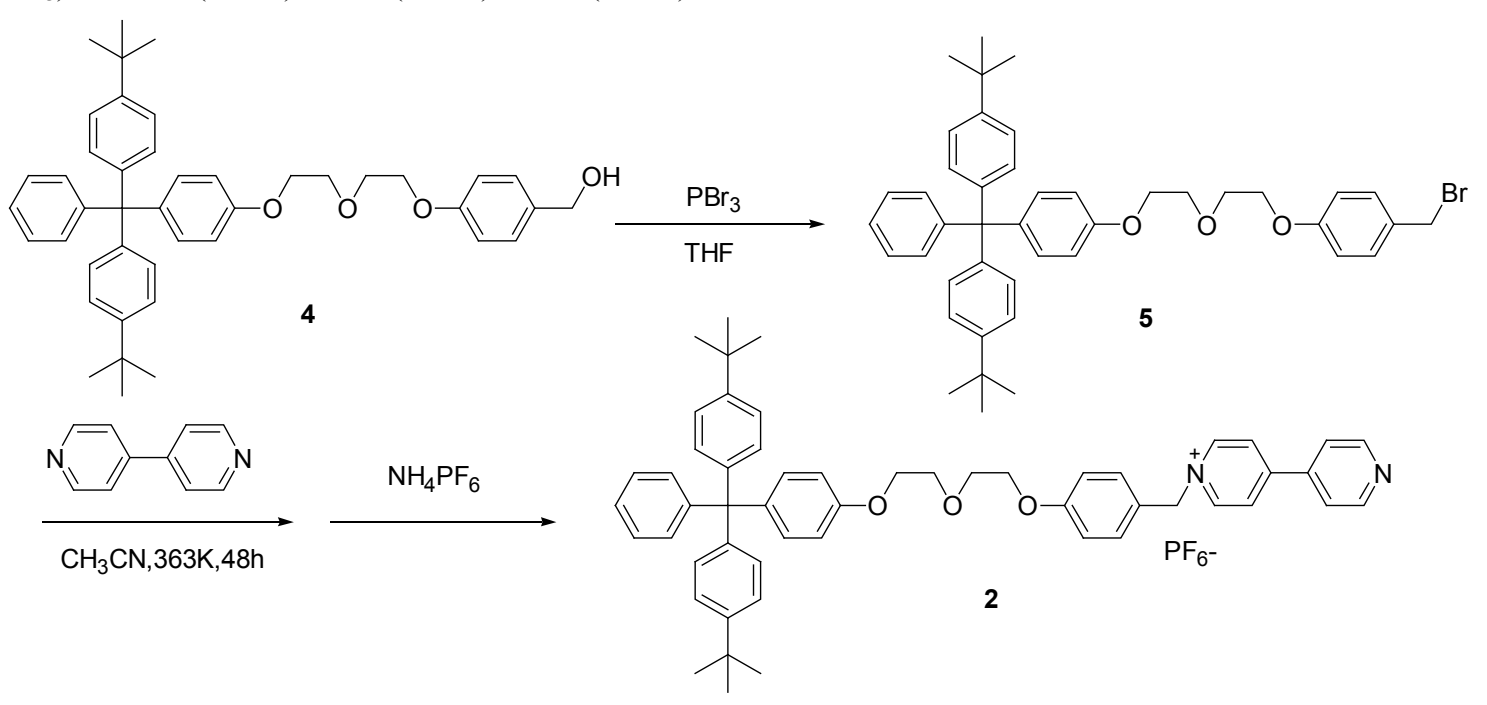

Scheme 3 


\section{References}

[1] (a) Schalley, C. A.; Beizai, K.; Vogtle F. Acc. Chem. Res. 2001, 34, 465.

(b) Collier, C. P.; Wong, E. W.; Belohradsky, M.; Raymo, F. M.; Stoddart, J. F.; Kuekes, P. J.; Williams, R. S.; Heath, J. R. Science 1999, 285, 391.

(c) Feng, M.; Guo, X. F.; Lin, X.; He, X. B.; Ji, W.; Du, S. X.; Zhang, D. Q.; Zhu, D. B.; Gao, H. J. J. Am. Chem. Soc. 2005, 27, 15338 .

(d) Balzani, V.; Credi, A.; Raymo, F. M.; Stoddart, J. F. Angew. Chem., Int. Ed. 2000, 39, 3348.

(e) Zheng, B.; Zhang, M.; Dong, S. Y.; Liu, J.; Huang, F. H. Org. Lett. 2012, 14, 306.

(f) Yamada, Y.; Mihara, N.; Shibano, S.; Sugimoto, K.; Tanaka, K. J. Am. Chem. Soc. 2013, 135, 11505.

(g) Shen, J. F.; Yu, X. T.; Ye, Y. Y.; Chen, R. E.; Jiang, H. J.; Zhou, Q. Z. Chin. J. Org. Chem. 2012, 32, 2265 (in Chinese) (沈健芬, 于雪涛, 叶余原, 陈仁尔, 蒋华江, 周其忠, 有机化学, 2012, 32, 2265.)

(h) Xue, W.; Li, Z. Y.; Liu, G. X.; Chen, X. Q.; Li, T. T.; Liu, S. H.; Yin, J. Org. Biomol. Chem. 2014, 12(27), 4862.

[2] (a) Ballardini, R.; Balzani, V.; Credi, A.; Gandolfi, M. T.; Venturi, M. Acc. Chem. Res. 2001, 34, 445

(b) Li, Z. T. Chin. J. Org. Chem. 2000, 20, 655 (in Chinese).

(黎占亭, 有机化学, 2000, 20, 655.)

(c) Li, H.; Xu, X. C.; Chen, J. W.; Yang, C. L.; Qin, J. G. Chin. J. Org. Chem. 2008, 28, 2057 (in Chinese).

(李吴，许曦晨，陈嘉伟，杨楚罗，秦金贵，有机化学，2008，28， 2057.)

(d) Liu, P.; Shao, X. G.; Cai, W. S. Prog. Chem. 2013, 25(5), 692 (in Chinese).

(刘鹏, 邵学广, 蔡文生, 化学进展, 2013, 25(5), 692.)

[3] (a) Ji, F. Y.; Zhu, L.-L. Chin. J. Org. Chem. 2011, 31, 973 (in Chinese).

(纪奉元, 朱亮亮, 有机化学, 2011, 31, 973.) (b) Zhai, C. X.; Huang, F. H. Sci. China Chem. 2009, 39, 315 (in Chinese).

(翟春熙, 黄飞鹤, 中国科学: 化学, 2009, 39, 315.)

[4] Chambron, J. C.; Heitz, V.; Sauvage, J. P. J. Chem. Soc., Chem. Commun. 1992, 1131.

[5] Anelli, P. L.; Ashton, P. R.; Ballardini, R.; Balzani, V.; Delgado, M.; Gandolfi, M. T.; Goodnow, T. T., Kaifer, A. E.; Philp, D.; Pietraszkiewicz, M.; Prodi L.; Reddington, M. V.; Slawin, A. M. Z.; Spencer, N.; Stoddart, J. F.; Vicent, C.; Williams, D. J. J. Am. Chem. Soc. 1992, 114, 193.

[6] Ashton, P. R.; Ballardini, R; Balzani, V.; Lohradsky, M.; Gandolfi, M. T.; Philp, D.; Prodi, L.; Raymo, F. M.; Reddington, M. V.; Spencer, N.; Stoddart, J. F.; Venturi, M.; Williams, D. J. J. Am. Chem. Soc. 1996, 118, 4931.

[7] Chiu, C. W.; Lai, C. C.; Chiu, S. H. J. Am. Chem. Soc. 2007, 129, 3500 .

[8] (a) Reuter, C.; Wienand, W.; Hübner, G. M.; Seel, C.; Vögtle, F. Chem. Eur. J. 1999, 5, 2692.

(b) Seel, C.; Vögtle, F. Chem. Eur. J. 2000, 6, 21.

[9] Hirose, K.; Nishihara, K.; Harada, N.; Nakamura, Y.; Masuda, D.; Araki, M.; Tobe, Y. Org. Lett. 2007, 9, 2969.

[10] Winn, J.; Pinczewska, A.; Goldup, S. M. J. Am. Chem. Soc. 2013, 135, 13318.

[11] Feng, Y. H.; Li, J. N.; Jiang, L. S.; Gao, Z. H.; Huang, W. C.; Jiang, F.; Luo, N. H.; Han, S. J.; Zeng, R. H.; Yang, D. Q. Eur. J. Org. Chem. 2011, 3, 562 .

[12] Chen, M.-J.; Zhou, S.-G.; Li, M.; Lei, C.-Y.; Xiao, R,; Jiang, L.-S. Chem. Res. Chin. Univ. 2013, 29, 1094.

[13] (a) Loeb, S. J.; Wisner, J. A. Angew. Chem., Int. Ed. 1998, 37, 2838 .

(b) Ashton, P. R.; Ballardini, R.; Balzani, V.; Credi, A.; Dress, K. R.; Ishow, E.; Kleverlaan, C. J.; Kocian, O.; Preece, J. A.; Spencer, N.; Stoddart, J. F.; Venturi, M.; Wenger, S. Chem. Eur. J. 2000, 6, 3558 .

[14] Jiang, L. S.; Okano, J.; Orita, A.; Otera, J. Angew. Chem., Int. Ed. 2004, 43, 2121. 